\title{
AISLAMIENTO DE HONGOS ENTOMOPATÓGENOS EN SUELO Y SOBRE GALLINAS CIEGAS (COLEOPTERA: MELOLONTHIDAE) EN AGROECOSISTEMAS DE MAÍZ
}

\author{
V. Manuel Hernández-Velázquez, ${ }^{1}$ Zito Cervantes Espíndola, ${ }^{2}$ Francisco J. \\ Villalobos, ${ }^{2}$ Laura Lina García ${ }^{1} \&$ Guadalupe Peña Chora ${ }^{3}$ \\ ${ }^{1}$ Centro de Investigación en Biotecnología (CEIB), ${ }^{2}$ Facultad de Ciencias Agropecuarias y ${ }^{3}$ Centro de \\ Investigaciones Biológicas (CIB). Cuerpo Académico de Entomología y Fitopatología. Universidad \\ Autónoma del Estado de Morelos (UAEM) Av. Universidad 1001, Col. Chamilpa, Cuernavaca, \\ Morelos, C. P 62210 México. Fax: (777) 3297030. \\ <vmanuelh@buzon.uaem.mx>
}

Hernández-Velázquez V. M., Z. Cervantes Espíndola, F. J. Villalobos; L. L. García \& G. Peña Chora. 2011. Aislamiento de hongos entomopatógenos en suelo y sobre gallinas ciegas (Coleoptera: Melolonthidae) en agroecosistemas de maíz. Acta Zoológica Mexicana (n. s.), 27(3): 591-599.

RESUMEN. En México se conoce poco sobre la presencia y abundancia de enemigos naturales de plagas suelo como las Phyllophaga spp. Por lo anterior se realizó el presente trabajo con los objetivos de aislar, identificar y conservar entomopatógenos en suelos cultivados con maíz y sobre inmaduros de gallina ciega en el estado de Morelos. En altitudes de 1000 a $2300 \mathrm{msnm}$, se realizaron cuarenta y cuatro colectas de suelo en sitios georeferenciados (GPS). La detección de hongos se realizó mediante la técnica de insecto trampa, Galleria mellonella (Lepidoptera: Pyralidae). Además, se colectaron inmaduros de gallina ciega de diversos estadios larvales en diferentes localidades, se separaron por morfoespecie de acuerdo a la forma del ráster y se tomaron fotografías de cada morfoespecie. Tanto de las larvas de G. mellonela como de las gallinas ciegas se realizaron aislamientos e identificación de hongos entomopatógenos. Se identificaron siete morfoespecies y tres especies de Phyllophaga, alimentándose sobre raíces de plantas de maíz, además de tres especies de Paranomala. Se conservan en la Colección del Laboratorio de Control Biológico del Centro de Investigación en Biotecnología de la Universidad Autónoma del Estado de Morelos (con respaldo en la Colección de Hongos Entomopatógenos del CNR$\mathrm{CB}$ ) cuarenta y ocho aislamientos de hongos entomopatógenos (siete aislados de gallina ciega y 41 de larvas de G. mellonella). De éstos, 15 corresponden a Metarhizium anisopliae, 30 a Beauveria bassiana y tres a Paecilomyces sp. Estos aislamientos poseen un gran valor para la zona, desde el punto de vista de conservación de la biodiversidad de enemigos naturales de plagas agrícolas y por su potencial como agentes de control biológico de GC y otras plagas del maíz.

Palabras clave: gallina ciega, morfoespecies, maíz, Galleria mellonella Beauveria bassiana, Metarhizium anisopliae.

Recibido: 17/01/2011; aceptado: 28/06/2011. 
Hernández-Velázquez V. M., Z. Cervantes Espíndola, F. J. Villalobos; L. L. García \& G. Peña Chora. 2011. Entomopathogenic fungi isolated from soil and immature white grubs (Coleoptera: Melolonthidae) in corn agrosystems. Acta Zoológica Mexicana (n. s.), 27(3): 591-599.

ABSTRACT. The knowledge on the presence and abundance of natural enemies of white grubs in Mexico is limited. Therefore the aim of the present work is to isolate, identify and preserve entomopathogens from corn cultivated soils and from collections made directly on white grubs in Morelos. Altitudes of these georeferenced (GPS) sites ranged between 1000 to 2300 masl. Forty four soil sample collections were made in these sites. Fungal detection was made by the trap insect technique using Galleria mellonella (Lepidoptera: Pyralidae) as a bait. Besides, white grub species present in each site belonging to different larval instars. These larvae were identified to morphospecies on the basis of the raster palidia and were observed until death or adult emergence. Field collected dead larvae with fungal infections were also identified in the same way. Both the larvae of $G$. mellonela and white grubs were used to isolate and identify the fugal species present. Fungal identification was made on the basis of morphology and fungal development on the insect which correlates with conidial size and shape and the conidial formation in colonies of purified isolates. Fourty eight fungal isolates (seven from white grubs and 41 G. mellonella) have been preserved in the Biological Control Laboratory of the CEIB-UAEM (a replicate has been placed in Fungal Entomopathogens Colllection of CNRCB). In this collection 15 strains of Metarhizium anisopliae, 30 strains of Beauveria bassiana and three strains of Paecilomyces sp. are present. This fungal diversity is valuable in the zone for conservation purposes and for its potencial as biocontrol agents against white grubs and other corn pests.

Key words: withe grubs, corn, Beauveria bassiana, Metarhizium anisopliae, entomopathogenic fungi.

\section{INTRODUCCIÓN}

En México, las especies de Phyllophaga (Coleoptera: Melolonthidae) conocidas comúnmente como "gallinas ciegas" (GC), representan un serio problema como plagas del suelo en varias regiones del país ya que se alimentan de las raíces (rizófagas) de diversos cultivos, reportándose los daños más importantes en maíz (Morón \& Rodríguez del Bosque 2010) Debido al hábito subterráneo durante su desarrollo larval, las GC son susceptibles a la infección por microorganismos como virus (Glare 1992), bacterias (Milner 1981, Klein \& Kaya 1995, Bixby et al. 2007), protistas (Brooks 1988, Jackson 2006), hongos (Ferron 1978, Jackson 2006) y nemátodos (Klein 1990, Kopenhöfer et al. 2004, Kopenhöfer et al. 2006); teniendo éstos un alto potencial para ser utilizados en el control microbiano por incremento que es una de las estrategias de control biológico de plagas aplicada en el control microbiano.

Una estrategia ecológicamente segura para utilizar entomopatógenos en el combate de plagas de suelo, es basarse en la identificación del complejo de especies plaga y sus patógenos nativos para posteriormente seleccionar el microorganismo con mayor potencialidad, tomando como criterios de referencia la virulencia, movilidad, persistencia, especificidad y los costos de producción del patógeno (Villalobos 1992).

Las condiciones de humedad y temperatura relativamente estables, así como la protección del suelo contra la luz ultravioleta, favorecen la infección de larvas de melolóntidos por hongos entomopatógenos (Villani et al. 1992) y los convierten en un grupo con alto potencial como agentes de control microbiano sobre larvas rizó- 
fagas. En este sentido, se han dado a conocer 25 especies de hongos patógenos de GC; sin embargo, la información tanto del patógeno como del huésped es frecuentemente incompleta o deficiente, ya que los hongos son descritos como procedentes de "Coleoptera" o "escarabajo" (Hernández et al. 2010). En México, los hongos patógenos de melolóntidos han sido poco estudiados, reportándose para Metarhizium anisopliae (Metchnikoff) Sorokin, Beauveria bassiana (Bálsamo) Vuillemin y Cordyceps sp. (Villalobos 1992, Hernández-Velázquez et al. 1996) como patógenos de especies de Phyllophaga. Actualmente en la Colección de Hongos Entomopatógenos del Centro Nacional de Referencia de Control Biológico se conservan 60 aislamientos de M. anisopliae y B. bassiana purificados de GC (Hernández et al. 2010).

Los hongos entomopatógenos presentan un amplio espectro de huéspedes (De Faria \& Wraight 2007), intervalo geográfico y potencial para el control de especies de Phyllophaga; por estas razones es importante colectar, purificar y conservar el germoplasma de la mayor variedad de sitios y especies. Al igual que con otros organismos, la conservación de cepas en colecciones de referencia deben ser prioritarios, ya que algunos genotipos pudieran perderse debido a cambios ambientales locales. En México se conoce poco sobre la presencia y abundancia de hongos entomopatógenos en suelos agrícolas y sobre GC. Por ello se realizó el presente trabajo con los objetivos de aislar, identificar y conservar hongos entomopatógenos en suelos cultivados con maíz y sobre GC en el Estado de Morelos.

\section{MATERIALES Y MÉTODOS}

Aislamiento de hongos de muestras de suelo. Se realizaron colectas de suelo en áreas predeterminadas por altitud (de 1000 a $2300 \mathrm{msnm}$ ) y temperatura media anual (de 14 a $24{ }^{\circ} \mathrm{C}$ ) en sustratos con cultivo o con antecedente de haber sido cultivados con maíz en el ciclo anterior; además, en todos los sitios se tomó una muestra de suelo en áreas no cultivadas cercanas a la parcela con cultivo de maíz. Los sitios muestreados fueron ubicados con Geoposicionador Satelital (Garmin etrex), con los siguientes datos: número de colecta, nombre del colector, fecha de colecta, planta hospedera y localización geográfica (Estado y municipio, coordenadas, msnm). Las muestras de suelo fueron colectadas de la siguiente forma: en una superficie de $10 \mathrm{~m}^{2}$ se marcaron tres puntos en los cuales se colectó una muestra de los primeros $15 \mathrm{~cm}$ de suelo (aproximadamente $1 \mathrm{~kg}$ ) la cual se transportó al laboratorio en bolsas de plástico de $2 \mathrm{~kg}$ de capacidad, debidamente etiquetadas, dentro de hieleras con la finalidad de evitar temperaturas altas. Las muestras se conservaron en cuarto frío a $1{ }^{\circ} \mathrm{C}$ por no más de dos meses, hasta su procesamiento. Para la detección y aislamiento de hongos entomopatógenos se utilizó la técnica del insecto trampa (Zimmermman 1986) con larvas del "gusano de la cera" Galleria mellonella (Lepidoptera: Pyralidae) reproducidas en laboratorio. De cada muestra de suelo homogenizada, $500 \mathrm{~g}$ se colocaron 
en un recipiente plástico de $14 \times 18 \times 6 \mathrm{~cm}$ junto a siete larvas de $G$. mellonella de último estadio y fueron incubadas a $27+1{ }^{\circ} \mathrm{C}$ durante 7 días. Posteriormente, las larvas muertas con síntomas de infección por hongos se colocaron en cámara húmeda. El aislamiento y purificación de los hongos se realizó en cámara de flujo laminar por transferencia directa de conidios y/o micelio a tubos de ensayo con medio nutritivo a base de sabouraud, agar, dextrosa y cloramfenicol (antibiótico). La identificación se realizó con base en la coloración y crecimiento de los hongos sobre los insectos y se corroboró en microcultivos y observaciones en microscopio con contraste de fases, siguiendo las claves de Humber (1997).

Los datos obtenidos del porcentaje de insectos trampa infectados por hongos por sitio se transformaron vía arcoseno de $\sqrt{ }_{\mathrm{y}_{\mathrm{i}}}$, antes y después de ser transformados, se realizó una prueba de normalidad y homogeneidad de varianzas (SAS 2002) cuando los datos tuvieron una distribución normal fueron analizados mediante pruebas de t $(\alpha 0.05)$ o con la prueba Kruskal-Wallis cuando presentaron una distribución no normal (Zar 1999), comparando el porcentaje de insectos con hongos en suelos cultivados y no cultivados.

Obtención de hongos a partir de GC colectadas. Para obtener hongos entomopatógenos directamente de GC se colectaron $\sim 20$ larvas de diversos estadios por localidad en catorce diferentes localidades, se trasladaron al laboratorio en forma individualizada en recipientes de plástico, se conservaron en suelo del sitio de colecta alimentándolas con trozos de zanahoria, a temperatura de $27 \pm 1{ }^{\circ} \mathrm{C}$ y se separaron por morfoespecie de acuerdo a la forma del ráster (Aragón \& Morón 2004, RamírezSalinas et al. 2004). Se tomaron fotografías de cada morfoespecie y se mantuvieron en observación en forma individual hasta que muriera la larva o emergiera el adulto; las larvas que se colectaron muertas en campo fueron separadas e identificadas de la misma manera. Se realizaron aislamientos e identificación de las especies de hongos de acuerdo a la metodología descrita anteriormente; se registró además, la morfoespecie y desarrollo del insecto.

\section{RESULTADOS Y DISCUSIÓN}

Se colectaron cuarenta y cuatro muestras de suelo, veintidós en cultivo de maíz y veintidós en áreas no cultivadas cercanas; el $68.18 \%$ de las muestras fueron positivas a la presencia de B. bassiana, M. anisopliae y Paecilomyces spp. De las cuarenta y cuatro muestras, $B$. bassiana fue detectado en el $60.8 \%$, M. anisopliae en el $21.7 \%$, y Paecilomyces spp. en el $6.5 \%$. Al comparar el porcentaje de insectos infectados por género de hongo, se observan diferencias significativas ( $p<0.0115$, Kruskal-Wallis), separando a $B$. bassiana como la especie que infectó un mayor número de insectos trampa, mientras que $M$. anisopliae y Paecilomyces spp. son estadísticamente iguales en cuanto a este parámetro (Fig. 1). Resultados similares han sido reportados por 


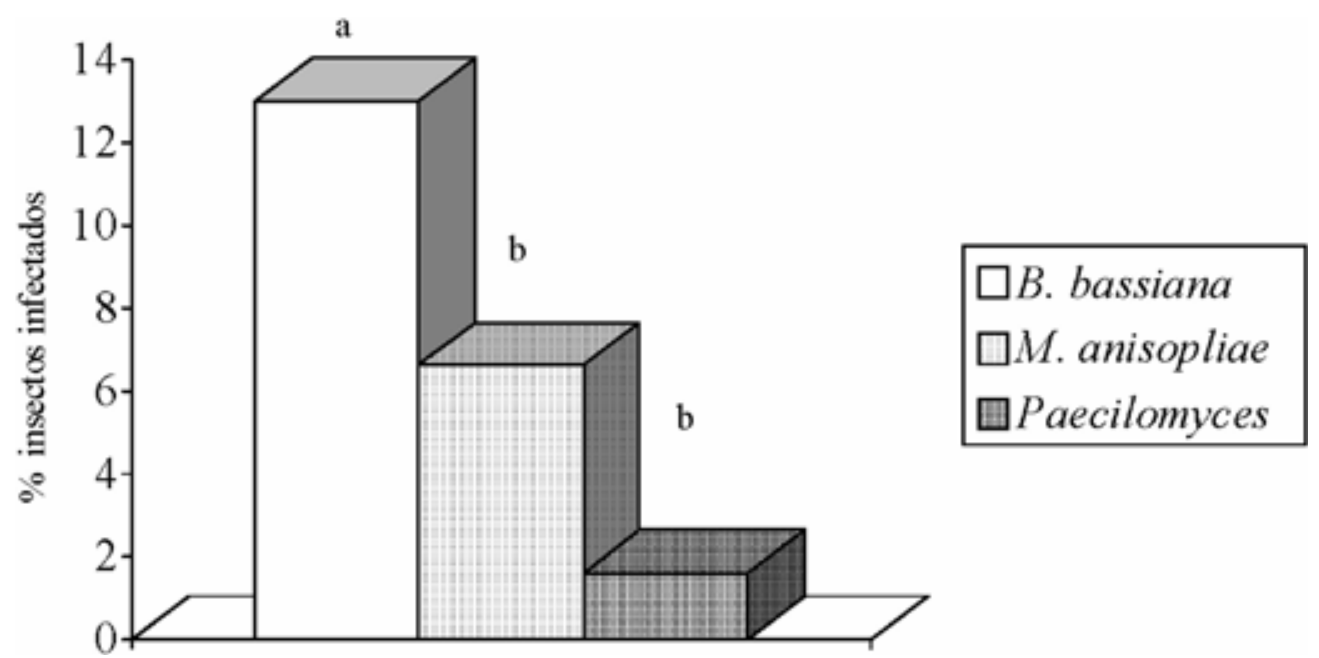

Figura 1. Porcentaje de larvas de Galleria mellonella infectadas (media \pm EE) por, Beauveria bassiana (13.0 \pm 1.9$)$ Metarhizium anisopliae (6.6 \pm 1.8$)$ y Paecilomyces sp. (1.6 \pm 1.0$)$ en suelo de 22 localidades del Estado de Morelos. La letras indican diferencia entre las medias de las poblaciones (Prueba Kruskal-Wallis $p=0.0132$ ).

otros autores para México y Canadá (Bidochka et al. 1998, Lezama-Gutiérrez et al. 2001 y Molina-Ochoa et al. 2002). Bidochka y colaboradores (1998) reportaron la presencia de los mismos géneros de hongos entomopatógenos en suelos de Canadá, aunque en porcentajes más altos, pero también predominando $B$. bassiana. Por otra parte en México, en muestras de suelo de los estados de Colima, Michoacán, Nayarit, Veracruz, Jalisco y Sinaloa, no se encontró a Paecilomyces y M. anisopliae fue la especie dominante (Molina-Ochoa et al. 2002). Lezama-Gutiérrez y colaboradores (2001) también reportaron a $M$. anisopliae con mayor abundancia en muestras de suelo de Michoacán, Colima, Jalisco y Tamaulipas. Esto es importante de resaltar, ya que en los sitios en los cuales se ha reportado a $M$. anisopliae con mayor frecuencia en México son zonas costeras tropicales en las cuales la humedad relativa y la temperatura son más altas. Asimismo, se conoce que B. basssiana sobrevive por más tiempo en áreas de clima templado y donde existen huéspedes para tener continuidad en su ciclo de infección (Vanninen 1996).

El porcentaje de insectos infectados por hongos por sitio fue mayor y estadísticamente diferente ( $p=0.0115$, Kruskal-Wallis) en suelos no cultivados $(27.26 \%)$ con respecto a los suelos cultivados con maíz (12.26\%) (Fig. 2). En este sentido, Sosa-Gómez \& Moscardi (1994) reportan una mayor prevalencia de B. bassiana, $M$. ansisopliae, Nomuraea rileyi y Paecilomyces sp. en suelos no cultivados con respecto 
b

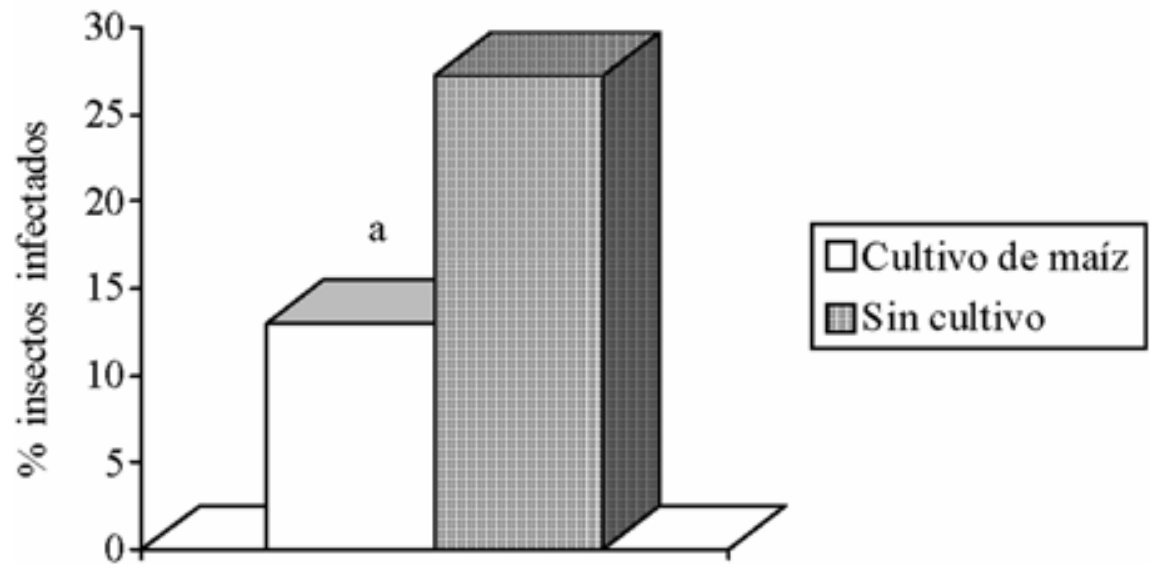

Figura 2. Porcentaje de larvas de Galleria mellonella infectadas (media \pm EE) por hongos entomopatógenos en suelo de 22 localidades del Estado de Morelos en suelos cultivados con maíz $(12.98 \pm 3.92)$ y sin cultivados $(27.26 \pm 3.92)$. Letras diferentes indican diferencia entre las medias de las poblaciones (Prueba Kruskal-Wallis $p=0.0132$ ).

a los cultivados con soya. Lo anterior tal vez se deba en gran parte a que en áreas de cultivo los conidios de los hongos son expuestos en mayor cantidad a la luz ultravioleta y temperaturas altas, los cuales tienen mayor influencia en la sobrevivencia de estos microorganismos.

El hongo entomopatógeno M. anisopliae fue aislado e identificado en cinco sitios, de 14 muestreados (Cuadro 1), sobre larvas de Paranomala sp. 1 y 2, Phyllophaga sp. 3 y 4 y sobre larvas de Cetoninae; asimismo $B$. bassiana fue detectado en dos sitios sobre Ligyrus sp. y Paranomala sp.1 (Cuadro 2). Los aislamientos de estos hongos se conservan actualmente en medio nutritivo a $6{ }^{\circ} \mathrm{C}$. Por otra parte, también se observaron dos larvas, una de $P$. obsoleta y la otra de $P$. sp. 3 (sitios F y M, respectivamente, Cuadro 2), con síntomas de infección de enfermedad lechosa y una larva de Paranomala sp. 2 con la enfermedad ámbar, todas de tercer instar.

Se conservan en la Colección del Laboratorio de Control Biológico del CEIBUAEM (con respaldo en la Colección de Hongos Entomopatógenos del Centro Nacional de Referencia de Control Biológico SAGARPA-SENASICA-DGSV) treinta y seis aislamientos de hongos entomopatógenos, dieciséis de GC, veinte de larvas de $G$. mellonella, catorce de M. anisopliae, veintiuno de B. bassiana y uno de Paecilomyces sp. Estos aislamientos son de un gran valor desde el punto de vista de conservación de la biodiversidad para la zona y se constituyen además como potenciales agentes de control biológico de GC y otras plagas de maíz. 
Cuadro 1. Sitios de colecta de gallinas ciegas en cultivo de maíz en el estado de Morelos.

\begin{tabular}{cllc}
\hline Colecta & \multicolumn{1}{c}{ Localidad } & \multicolumn{1}{c}{ Ubicación geográfica } & altitud (msnm) \\
\hline A & Tetela del Volcán & N 18.89462; WO 98.73398 & 2229 \\
B & Tetela del Volcán & N 18.88216; WO 98.73398 & 2115 \\
C & Jumiltepec & N 18.88483; WO 98.77992 & 1866 \\
D & Santiago Ocuituco & N 18.88060; WO 98.80215 & 1793 \\
E & Tlayca & N 18.70045; WO 98.84995 & 1137 \\
F & Axochiapan & N 18.49893; WO 98.84143 & 1315 \\
G & Tepalcingo & N 18.59101; WO 98.84143 & 1156 \\
H & Tetelilla & N 18.66014; WO 98.79218 & 1275 \\
I & Jumiltepec & N 18.88482; WO 98.77992 & 1869 \\
J & Metepec & N 18.88400; WO 98.73950 & 2100 \\
K & Santiago & N 18.87798; WO 98.80170 & 1794 \\
L & Totolapan & N 18.97429; WO 98.91369 & 1874 \\
M & San Andrés de la Cal & N 18.95696; WO 99.11691 & 1530 \\
N & Buenavista del Monte & nd & 1869 \\
\hline
\end{tabular}

nd $=$ no disponible

Cuadro 2. Entomopatógenos detectados sobre gallinas ciegas en el estado de Morelos.

\begin{tabular}{cll}
\hline Sitio & \multicolumn{1}{c}{ Entomopatógeno } & \multicolumn{1}{c}{ Gallina ciega } \\
\hline A & Metarhizium anisopliae & Paranomala sp.1 (23 pali) \\
& Enfermedad ámbar & Paranomala sp.2 (12-13 pali) \\
B & Metarhizium anisopliae & Cetoninae \\
E & Metarhizium anisopliae & Paranomala sp.2 (12-13 pali) \\
F & Enfermedad lechosa & P. obsoleta \\
H & Beauveria bassiana & Ligyrus sp. \\
M & Enfermedad lechosa & Pyllophaga sp.3 (14 pali pequeños, palidia oval) \\
& Metarhizium anisopliae & Pyllophaga sp.3 (14 pali pequeños, palidia oval) \\
N & Metarhizium anisopliae & Pyllophaga sp.4 (13 pali cortos) \\
& Beauveria bassiana & Paranomala sp.1 (23 pali) \\
\hline
\end{tabular}

AGRADECIMIENTOS. Este trabajo es una contribución al proyecto PROMEP/103.5/04/2862: “Aislamiento, identificación y evaluación de hongos entomopatógenos de gallina ciega Phyllophaga spp. de Morelos." 
Hernández-Velázquez et al.: Hongos entomopatógenos en agrosistemas de maíz

\section{LITERATURA CITADA}

Aragón, A. \& M.A. Morón. 2004. Descripción de las larvas de tres especies de Phyllophaga (Coleoptera: Melolonthidae: Melolonthinae) del Valle de Puebla, México. Folia Entomologica Mexicana, 43: 295-306.

Bidochka, M., J., Kasperski \& G. Wild. 1998. Occurrence of the entomopathogenic fungi Metarhizium anisopliae and Beauveria bassiana in soils from temperate and near-northern habitats. Canadian Journal of Botany, 76: 1198-1204.

Bixby, A., S. R. Alm, K. Power, P. Grewal \& S. Jr. Swier. 2007. Susceptibility of four species of turfgrass-infesting scarabs (Coleoptera: Scarabaeidae) to Bacillus thuringiensis serovar japonensis strain Buibui. Journal of Economic Entomology, 100: 1604-1610.

Brooks, W. M. 1988. Entomogenous protozoa, pp. 1-149. In: C.M. Ignoffo (Ed.), CRC Handbook natural pesticides. Volume V Microbial insecticides. Part A Entomogenous protozoa and fungi. CRC Press Inc. Boca Raton, Florida, USA.

De Faria, M. R. \& S. P. Wraight. 2007. Mycoinsecticides and micoacaricides: a comprehensive list with worldwide coverage and internacional classification of formulation types. Biological Control, 43: 237-256.

Ferron, P. 1978. Biological control of insect pests by entomogenous fungi. Annual Review of Entomo$\log$, 23: 409-442.

Glare, T. 1992. Viral diseases of scarabs, pp. 21-32. In: T.A. Jackson \& T.R. Glare (Eds.), Use of pathogens in scarab pest management. Intercept, England.

Hernández-Velázquez, V., A. Berlanga Padilla, J. Pérez Domínguez \& E. Garza González. 1996. Aislamiento de hongos patógenos de plagas de suelo en Jalisco y Nayarit, pp: 5-7. In: Memoria del XIX Congreso Nacional de Control Biológico, Culiacán, Sin. p. 202.

Humber, R.A. 1997. Fungi: preservation of cultures, pp. 269-279. In: L. Lacery (Ed.). Manual of techniques in insect pathology. Academic Press 409 pp.

Jackson, T. A. 2006. Scarabs as pests: a continuing problem. Coleopterists Society, Monograph 5: 102-119.

Klein, M.G. 1990. Efficacy against soil-inhabiting insect pests, pp. 195-214. In: R. Gaugler \& H.K. Kaya (Eds.), Entomopathogenic nematodes in biological control. CRC Press. Boca Raton, Florida, USA.

Klein, M. G. \& H.K. Kaya. 1995. Bacillus and Serratia especies for scarab control. Memorias do Instituto Oswaldo Cruz, 90: 87-95.

Kopenhöfer, A. M., E. M. Fuzy, R. Crocker, W. Gelenter \& S. Polavarapu. 2004. Pathogenicity of Steinernema scarabaei, Heterorhabditis bacteriophaga and $S$. glaseri to twelve white grub species. Biocontrol Science and Technology, 14: 87-92.

Kopenhöfer, A. M., P.S. Grewar \& E.M. Fuzy. 2006. Virulence of entomophatogenic nematodes Heterorhabditis bacteriophaga, Heterorhabditis zealandica and Steirnenerma scabadaei against five grub species (Coleoptera: Scarabaeidae) of economic importance in turfgrass in North America. Biological Control, 38: 397-404.

Lezama-Gutíerrez, R., J. Ham, J. Molina-Ochoa, M. López-Edwards, A. Pescador-Rubio, M. Gonzalez Ramirez \& E. Styler. 2001. Occurrence of entomopathogens of Spodoptera frugiperda (Lepidoptera: Noctuidae) in the Mexican states of Michoacán, Colima, Jalisco and Tamaulipas. Florida Entomologist, 84: 23-30.

Milner, R. J. 1981. Identification of the Bacillus popilliae group of insect pathogens, pp. 45-49. In: H.D. Burges (Ed.), Microbial control of pests and plant diseases 1970-1980. Academic Press. England.

Molina-Ochoa, J., R. Lezama-Gutiérrez, M. González-Ramírez, M. López-Edwards, M. Rodríguez-Vega \& F. Arceo-Palacios. 2003. Pathogens and parasitic nematodes associated with po- 
pulations of fall armyworm (Lepidoptera: Noctuidae) larvae in México. Florida Entomologist, 86: 244-253.

Morón, M. A. \& L. A. Rodríguez del Bosque. 2010. Importancia, historia y retos, pp. 3-17. In: L. A. Rodríguez del Bosque \& M. A. Morón (Eds.). Plagas del suelo. Mundi-Prensa México.

Ramírez-Salinas, C., M. A. Morón \& A. E. Castro-Ramírez. 2004. Descripción de los estados inmaduros de tres especies de Anomala, Ancognatha y Ligyrus (Coleoptera: Melolonthidae: Rutelinae y Dynastinae) con observaciones de su biología. Acta Zoológica Mexicana (n. s.), 20: 67-82.

Rodríguez del Bosque, L. A. 1988. Phyllophaga crinita (Burmeister): historia de una plaga del suelo (1955-1988), pp: 53-79. In: M. A. Morón y C. Deloya (Eds.), Memoria III Mesa Redonda sobre plagas del Suelo. Sociedad Mexicana de Entomología, A. C., ICI de México e Instituto de Ecología, A. C. Michoacán, México.

SAS Institute. 2002. User's guide, version 9.1. SAS Institute, Cary, N.C.

Sosa-Gómez, D. R. \& F. Moscardi. 1994. Effect of till and no-till soybean cultivation on dynamics of entomopathogenic fungi in the soil. Florida Entomologist, 77: 284-287.

Vanninen, I. 1996. Distribution and occurrence of four entomopathogenic fungi in Finland: effect of geografical location, habitat type and soil type. Mycological Research, 100: 93-101.

Villalobos, F. J. 1992. The potential of entomopathogens for the control of white grub pests of corn in Mexico, pp. 253-260. In: T. A. Jackson \& T. R. Glare (Eds.), Use of pathogens in scarab pest management. Intercept. England.

Villani, M. G., S. R. Krueger \& J. P. Nyrop. 1992. A case study of the impact of the soil environment on insect/pathogen interactions: scarab in turfgrass, pp: 111-126. In: T. A. Jackson \& T. R. Glare (Eds.), Use of pathogens in scarab pest management. Intercept Limited, Andover.

Zar, H. J. 1999. Biostatistical Analysis. Fourth Edition. Prentice-Hall, Inc, Englewood Cliffs, New Jersey, USA.

Zimmermann, G. 1986. The "Galleria" bait method for detection of entomopathogenic fungi in soil. Journal of Applied Entomology, 102: 213-215. 